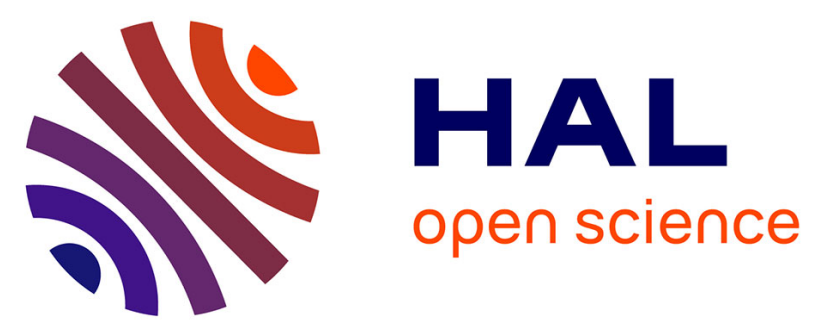

\title{
Chronic use of benzodiazepines and latent cognitive decline in the elderly: Results from the Three-city study.
} Thibault Mura, Cécile Proust-Lima, Tasnime N. Akbaraly, Hélène Amieva, Christophe Tzourio, Hugues Chevassus, Marie-Christine Picot, Hélène Jacqumin-Gadda, Claudine Berr

\section{To cite this version:}

Thibault Mura, Cécile Proust-Lima, Tasnime N. Akbaraly, Hélène Amieva, Christophe Tzourio, et al.. Chronic use of benzodiazepines and latent cognitive decline in the elderly: Results from the Three-city study.. European Neuropsychopharmacology, 2013, 23 (3), pp.212-23. 10.1016/j.euroneuro.2012.05.004 . inserm-00714718

\section{HAL Id: inserm-00714718 https://www.hal.inserm.fr/inserm-00714718}

Submitted on 5 Jul 2012

HAL is a multi-disciplinary open access archive for the deposit and dissemination of scientific research documents, whether they are published or not. The documents may come from teaching and research institutions in France or abroad, or from public or private research centers.
L'archive ouverte pluridisciplinaire HAL, est destinée au dépôt et à la diffusion de documents scientifiques de niveau recherche, publiés ou non, émanant des établissements d'enseignement et de recherche français ou étrangers, des laboratoires publics ou privés. 
Chronic use of benzodiazepines and latent cognitive decline in the elderly: results from the Three-city study

MURA Thibault ${ }^{1,2,3}$, M.D., PROUST-LIMA Cécile ${ }^{4,5}$, Ph.D., AKBARALY Tasnime ${ }^{1,6,7}$,

Ph.D., AMIEVA Hélène ${ }^{4,5}$, Ph.D., TZOURIO Christophe ${ }^{8}$ M.D., Ph.D., CHEVASSUS Hugues $^{2,3}$, PICOT Marie-Christine ${ }^{2,3}$, M.D., Ph.D., JACQUMIN-GADDA Hélène ${ }^{4,5}$, Ph.D., BERR Claudine ${ }^{1,7}$ M.D., Ph.D.

(1) INSERM, U1061, Neuropsychiatrie : Recherche Epidémiologique et Clinique; Université Montpellier I, 34093 Montpellier, Cedex 5, France (2) University Hospital of Montpellier, Medical Information Department, Clinical Investigation Center, 34093 Montpellier, France (3) INSERM, CIC 1001, Montpellier, France (4) INSERM U897, Centre de Recherche en Epidémiologie et Biostatistique, Bordeaux, F-33076 France (5) Université Victor Segalen Bordeaux 2, Bordeaux, F-33076 France (6) Department of Epidemiology and Public Health, University College London, WC1E 6BT London, United Kingdom E8 (7) CMRR Languedoc Roussillon, service de Neurologie, CHU Montpellier, 34093Montpellier, France (8) INSERM U708, 75651 Paris, Cedex 13, France

Submission to: the " European Journal of Neuropsychopharmacology"

Corresponding author and reprint requests: Dr. Thibault Mura

Thibault MURA, Centre d'Investigation Clinique 1001, Hôpital Saint Eloi, CHRU Montpellier, 80, avenue Augustin Fliche, 34000 Montpellier, France.

Tel: 0033467332328

Fax: 0033467332336

E-mail: t-mura@chu-montpellier.fr (email can not be published) 


\section{$\underline{\text { Abstract (250 words) }}$}

We aimed to examine whether long-term use of benzodiazepines is associated with an accelerated decline of cognitive performances by using a statistical model specifically adapted to multivariate longitudinal bounded quantitative outcomes. The data came from the "Threecity" study, a French population based study. All the subjects were 65 years old or older at inclusion and had been followed-up for 7 years. The use of benzodiazepines and cognitive functioning were assessed at each examination phase (baseline, 2, 4 and 7 years). Cognitive decline was analyzed using a nonlinear multivariate mixed model with a latent process. This model makes it possible to assess change over time of the latent cognitive process underlying several neuropsychological tests: Mini Mental Status Examination, Isaacs Set test, Benton Visual Retention Test, and Trail Making Test (A and B), and to describe and account for their metrological properties. Analyses were adjusted for age, center, gender, education, socioprofessional status, depression, insomnia, high blood pressure, hypercholesterolemia, alcohol, tobacco consumption and physical activity. 969 subjects who reported taking benzodiazepines for 2, 4 or 7 consecutive years were compared to 4226 subjects who were non benzodiazepine users. Chronic use of benzodiazepine was significantly associated with a lower latent cognitive level $(\beta=-1.79 \mathrm{SE}=0.25 \mathrm{p}=<0.001)$, but no association was found between chronic use and an acceleration of cognitive decline, neither on the latent cognitive process $\left(\beta^{*}\right.$ time $\left.=0.010 \mathrm{SE}=0.04 \mathrm{p}=0.81\right)$, nor on specific neuropsychological tests. Our results suggest that chronic benzodiazepine use is associated with poorer cognitive performance but not with accelerated cognitive decline with age.

$\underline{\text { Key words: }}$ cognitive decline; cognitive impairment; benzodiazepines; latent process; longitudinal study. 


\section{INTRODUCTION}

In Europe, benzodiazepines are among the most widely prescribed psychotropic drugs, especially in the elderly population in which benzodiazepines are used for extended periods of time compared to younger age ranges (Morgan et al, 1988; Ohayon and Lader, 2002). While the European authorities and Food and Drugs Administration have recommended limiting the duration of treatment to 4 months (Food and Drug Administration, 1980; Haute Autorité de Santé, 2007; National Health Service Grampian, 2008), some European studies have shown that almost $25 \%$ of elderly subjects reported daily use of benzodiazepines for more than 6 months (Lechevallier et al, 2003). Alterations in the pharmacokinetics and pharmacodynamics of benzodiazepines, combined with age-related decrease in the reserve of the central nervous system are liable to lead the elderly to be particularly sensitive to the cognitive side effects of benzodiazepines.

Although acute effects of occasional use of benzodiazepines on psycho-motor memory and attention performances have been demonstrated (Buffett-Jerrott and Stewart, 2002; Curran, 1991; Stewart, 2005), less is known regarding the effect of the long-term impact on cognitive outcome. Some studies have suggested a cross-sectional deleterious impact of longterm benzodiazepine use on memory performance (Bierman et al, 2007; Hanlon et al, 1998), fluid intelligence (Bierman et al, 2007) and global cognition (Paterniti et al, 2002), but the longitudinal effects of benzodiazepines on cognitive change with age (cognitive decline) are more difficult to assess and have led to conflicting results (Verdoux et al, 2005). Whereas one study highlighted accelerated cognitive decline (Paterniti et al, 2002), two others failed to find any association (Dealberto et al, 1997; Lagnaoui et al, 2009). The inconsistency of these findings may be due to difficulties in assessing adequately age-related cognitive change. Indeed, in these studies the use of arbitrary cut-offs to define subjects with or without cognitive decline may constitute a major limitation. Furthermore, statistical approaches using 
differences between two points when studying cognitive change may lead to biased results due to the metrological properties of neuropsychological tests not taken into account (Galasko et al, 2000; Proust-Lima et al, 2011) (i.e. ceiling effects, varying sensitivity to change, also called curvilinearity). The classical statistical alternative which consists in using a linear mixed model does not correct this bias.

In the present paper, we aimed to analyze the association between chronic benzodiazepine use and cognitive outcomes in subjects over 65 , by distinguishing the crosssectional effects of benzodiazepines on cognitive level, and the longitudinal effects on cognitive decline. To do this, we applied a nonlinear multivariate mixed model involving a latent cognitive process underlying several neuropsychological tests considered simultaneously and measured repeatedly over time. This model makes it possible to account for the metrological properties of tests (curvilinearity and ceiling/floor effects) when estimating the effects of a covariate, and distinguishes the global effect on cognition and the specific effects on the cognitive domains covered by neuropsychological tests (Proust et al, 2008; Proust et al, 2006). 


\section{EXPERIMENTAL PROCEDURES}

\section{Study Design \& Participants:}

Data came from the multi-site prospective "Three City Study" (E3C), a cohort study of 9,294 community-dwelling persons aged 65 years and over, recruited from the electoral rolls of three French cities between 1999 and 2001 (The 3C Study Group, 2003). The study protocol was approved by the Ethical Committee of the University-Hospital of Bicêtre (France) and written informed consent was obtained from each participant. The cohort was followed up at 2, 4 and 7 years with face-to-face interviews and medical examinations in a medical center or at home. Because the definition of chronic use of benzodiazepines required data on drug consumption from the baseline and 2-year follow up, we excluded subjects who (Figure 1): 1) (N=970) had reported a change in benzodiazepine use between the baseline and the 2-year follow up; 2) were dead $(\mathrm{N}=274)$ or missing $(\mathrm{N}=918)$ at the 2-year follow-up; or 3) $(\mathrm{N}=327)$ were diagnosed with prevalent or 2-year incident dementia cases. Subjects were not excluded when these events occurred at 4 or 7 years of follow-up. Among these 6,805 subjects, 212 who were not subjected to any measurement for each neuropsychological test during their follow-up, and 1,398 subjects with missing data for baseline adjustment covariates (listed in Table 1) were excluded from the analysis. The present report was finally based on 5,195 participants.

Compared to those excluded, the participants included in the present analysis were younger, had higher occupational grades, practiced more frequently regular physical activity, were less likely to report depressive or anxious symptoms and performed better in cognitive tasks at baseline. (Data not shown) 


\section{Data Collection}

Neuropsychological evaluation: The cognitive tests were administered by trained psychologists at baseline and repeated at the 2, 4 and 7-year follow-up visits.

- The Mini Mental State Examination (MMSE) (Folstein et al, 1975) was used as an index of global cognitive performance. The scores range from 0 to 30 .

- The Benton Visual Retention Test (BVRT) (Benton, 1965), which evaluates immediate visual memory, consists in the presentation of a stimulus card displaying a geometric figure for 10 seconds after which individuals are asked to identify the initial figure from 4 possibilities. Fifteen figures are successively presented and scores range from 0 to 15 .

- The Isaacs Set Test (IST) (Isaacs and Kennie, 1973) consisting in generating words belonging to 4 semantic categories (cities, fruits, animals and colors) in 15 seconds, measured mostly semantic verbal fluency abilities but also the speed of verbal production. The scores range from 0 to 66 .

- The Trail Making Test, forms A and B (TMT-A and TMT-B) (Reitan, 1958), evaluates attention, visuomotor processing speed and mental flexibility capacities. TMT-A assesses attention and visuomotor processing speed by requiring patients to connect numbered circles in sequence. TMT-B assesses attention and visuomotor processing speed, but also demands mental flexibility as it requires patients to connect circles by alternating numerical and alphabetical sequences. The average number of correct displacements in 10 seconds can be calculated for each subject ((number of correct items/total time)*10). The range observed varied from 0 to 13.4 for TMT-A, and from 0 to 7.8 for TMT-B.

Assessment of benzodiazepine use: At each phase of examination, an inventory of all drugs (prescription and over-the-counter drugs) taken more than once a week during the preceding month was included in a standardized interview. Medical prescriptions and, where feasible, the medications themselves were checked by the interviewer. No data on dose, duration of 
treatment or reason for prescribing the drug were collected. Drugs were recorded and coded according to the WHO Anatomical Therapeutic Chemical (ATC) classification system (WHO Collaborating Centre for Drug Statistics Methodology, 2000).

All classes of benzodiazepines were considered: anxiolytic (ATC code: N05BA), hypnotic and sedative (N05CD), antiepileptic (N03AE), and myorelaxing (M03BX07). Hypnotic drugs derived or connected with benzodiazepines (N05CF) were also considered. Participants who did not report taking benzodiazepines both at baseline and 2-year follow-up were classified as "non-users". Those who used benzodiazepines both at inclusion and at 2year follow-up were defined as “chronic users”. As previously described (figure 1), to avoid classification bias linked to punctual use, subjects who reported a change in benzodiazepine use between the baseline and the 2-year follow up ( $\mathrm{N}=970)$ were excluded from this analysis.

In order to minimize prescription bias and to limit the interference of acute effect in the longitudinal part of the analysis, cognitive data were censored at 4 and/or 7-year follow-up visits when a subject was diagnosed as demented, or when they reported a change in benzodiazepine use (new prescription or discontinuation).

Covariates: Socio-demographic and health behavior consisted of sex, educational level, socio-professional status, alcohol consumption, tobacco use and physical activity. Health status was ascertained using a number of measurements: glycaemia (normal: fasting glucose $<6.1 \mathrm{mmol} / 1$; high 6.1 to $7 \mathrm{mmol} / 1$; diabetes $>7 \mathrm{mmol} / 1$ or treated), cholesterol levels (Hypercholesterolemia: total cholesterol level $\geq 7.2 \mathrm{mmol} / \mathrm{l}$ or treated by lipid lowering agents) and ApoE genotyping, as described previously (Ritchie et al, 2007), hypertension (defined as having systolic blood pressure $>160 \mathrm{~mm} \mathrm{Hg}$ or diastolic blood pressure $>95 \mathrm{~mm}$ $\mathrm{Hg}$ or use of antihypertensive drugs), cardiovascular disease history (self-reported angina pectoris, myocardial infarction or cardio-vascular surgery), insomnia (at least two positive 
responses in the following issues: poor sleep; recurrent difficulty to fall asleep; frequent waking at night or in the morning; taking medication for this indication). Finally, depressive symptoms, assessed by the Center for Epidemiological Studies-Depression Scale (CES-D, cut-off point 17 for men, 23 for women) (führer R and Rouillon, 1989) or antidepressant use, and anxiety symptoms, assessed by the Spielberger inventory trait (we used the $75^{\text {th }}$ percentile as cut-off to define high anxiety: $>43$ for men and $>48$ for women), were also considered.

\section{Statistical analysis}

Baseline characteristics were compared using the Khi2 test for qualitative variables and Wilcoxon's non-parametric test for quantitative variables. To analyze the relationship between benzodiazepine use and cognitive trajectory, nonlinear mixed models with latent process for multivariate longitudinal data were used (Proust et al, 2006) (illustrated in figure 2). The statistical model assumes that the correlation between the five neuropsychological tests is induced by a latent cognitive process representing the common factor of the neuropsychological tests. The model is divided into two parts estimated simultaneously: (a) a linear mixed model describes change over time in the latent cognitive process and evaluates the cross-sectional and longitudinal common effects of covariates on this latent cognitive trajectory, and (b) test-specific measurement models link each administration of the neuropsychological tests with the latent cognitive process, by accounting for cross-sectional and longitudinal test-specific associations with covariates and metrological properties of the tests.

Effect of the covariates on the latent cognitive process (referred to as (a)). The evolution of the common latent cognitive process was modeled using a linear mixed model (Laird and Ware, 1982), which evaluates changes of a repeated outcome over time (i.e. the latent cognitive process) and accounts for correlation between the repeated measurements of 
each subject. The linear mixed model included a subject-specific random intercept and random coefficients for time after inclusion (in years of follow-up). A Brownian motion was added to account for individual deviations from this quadratic trend, thus relaxing the parametric form of the model. The time of first assessment of cognitive level for each patient was identified by a covariate and included in the model to adjust for learning effects (Jacqmin-Gadda et al, 1997).

Test-specific effects of the covariates (Contrasts) referred to as $(b)$ : The test-specific models defined flexible links between the neuropsychological tests and the latent cognitive process. The model assumed that a parameterized test-specific nonlinear transformation of each test was a noisy measurement of the latent cognitive process adjusted for covariates, time, and test- and subject-specific variability. This part of the model also evaluated the specific effect of the covariate on each test after adjustment for the latent cognitive process and the common effect of the covariate on it. Thus, these test-specific effects, called contrasts, were used to evaluate whether the covariate had a different specific impact on each neuropsychological test in addition to its global effect on the latent cognitive process, and quantify this differential impact.

Overall effect on each specific test (referred to as (c)): the (cross-sectional or longitudinal) overall effect of a covariate on each specific test was calculated by adding together the effect of the covariate on the latent cognitive process (a) and the test-specific effect (contrast) (b).

The nonlinear test-specific transformations that link each neuropsychological test with the latent cognitive process were used to account for the global metrological properties of the tests (Proust-Lima et al, 2007). These transformations are covariate-and-time-invariant parametric functions depending on parameters that are estimated simultaneously with the other parameters of the model. Beta cumulative distribution functions were chosen as flexible 
transformations. These functions offered a large variety of shapes (concave, convex, sigmoid, or simply linear) and thus accounted for the curvilinearity of the tests. The latent cognitive process was defined in $[0,100]$ where 0 and 100 corresponded respectively to the minimal and maximal latent cognitive level. The complete methodology was detailed elsewhere (Proust-Lima et al, 2008; Proust et al, 2006).

All models were adjusted for the age of the participants at inclusion in the study, and its interaction with time after inclusion. Covariates marginally associated $(\mathrm{p}<0.05)$ with chronic use of benzodiazepines in a univariate analysis were considered to be potential confounders of the benzodiazepine-cognition relationship and were first included in the multivariate analyses. Only covariates associated with latent cognitive level or latent cognitive change were finally retained in the model. Further analyses were conducted to assess the effects of benzodiazepines according to their different categories according to ATC classification. Similar analyses were repeated by distinguishing anxiolytic benzodiazepines (ATC Code: N05BA) from sedative or hypnotic benzodiazepines (N05CD or N05CF).

Due to missing data (1,034 subjects without anxiety score), the main analyses were performed without adjustment for anxiety. However, sensitivity analyses were performed, with this adjustment, in order to exclude any confounding bias due to this covariate. Statistical tests were performed at the conventional two-tailed $\alpha$ level of 0.05 . Data were analyzed using SAS (Cary, North Carolina) statistical software and a FORTRAN90 executable for the nonlinear mixed model with latent process (program NLMULTIMIX available at http://biostat.isped.u-bordeaux2.fr/). 


\section{RESULTS}

\section{Characteristics of participants:}

969 of the 5,195 participants included in the present analyses were chronic benzodiazepine users. The mean age was 73.47 years old $(\mathrm{SD}=5.1)$ with women making up $59.9 \%$ of the sample. At baseline, $69.9 \% \quad(n=677)$ of chronic users were taking benzodiazepines classified as anxiolytic, $7.4 \%(n=72)$ as sedatives, $27.0 \%(n=262)$ as hypnotic drugs derived or connected with benzodiazepines, $4.1 \%(\mathrm{n}=40)$ as antiepileptic, and $2.0 \%(\mathrm{n}=19)$ as myorelaxing.

The characteristics of the participants as a function of chronic benzodiazepine are detailed in Table 1. Compared to non users, chronic users were more likely to be women, older, and less likely to have higher level of education or occupational grade, smoke, or practice regular physical activity. Chronic users were more likely to report cardiovascular antecedents, depressive symptoms, and have hypertension or hypercholesterolemia. Chronic users also presented significantly lower cognitive performances at baseline compared to nonusers whatever the cognitive test considered.

\section{Cognitive latent process modeling:}

The estimated transformation between each test and the latent cognitive process are displayed in figure3. Except for IST, transformations indicate nonlinear relationships between neuropsychological tests and the latent process. For MMSE, Figure 3 shows that the maximum value of the test was reached for a value of the latent process (approximately 85) lower than its maximum (100), indicating a strong ceiling effect. Furthermore, the concave shape of the MMSE transformation shows that a decline of MMSE does not correspond to the same intensity of decline of the latent process over the whole range of the test. Indeed, a loss 
of 10 points in the latent cognitive process scale between 70 and 60 represents a loss of 1 MMSE point, whereas the same loss between 40 and 30 corresponds to a loss of 3 MMSE points, suggesting that: 1) changes in MMSE cannot be interpreted without accounting for the initial value, and 2) MMSE is more appropriate for detecting decline in individuals with low or moderate cognitive levels rather than in individuals with a high cognitive level.

The BVRT shape is also concave with a smaller ceiling effect. The shape of IST is quite linear, revealing that a decline in this test represents roughly the same intensity of decline for the latent process whatever the initial cognitive levels. TMTA and TMTB present convex shapes, indicating that these tests are more sensitive to cognitive changes in high levels of cognition than in low ones. A considerable floor effect is observed for TMTB.

\section{Association between chronic benzodiazepine use and cognitive trajectories:}

The results from the nonlinear mixed model with a latent cognitive process (Table 2) showed that chronic benzodiazepine use was significantly associated with cross-sectional lowering of the latent cognitive level both in crude analyses (benzodiazepines=-2.51 pvalue $<0.001$ ) and in adjusted analyses (benzodiazepines $=-1.79$ p-value<0.001), (Part (a)Table 2). As shown by test-specific contrasts (Part (b)-Table 2), this cross-sectional association between benzodiazepines and lower cognitive performance was significantly more pronounced for immediate visual memory (BVRT) and for attention and visuomotor processing speed (TMT-A), but significantly less so on the global cognitive functioning measured by MMSE. The overall cross-sectional effect of benzodiazepines (c) on each specific test, estimated by adding together the global effect (a) and the test-specific effects (contrast) (b), are presented in Figure 4 and show that chronic benzodiazepine use was significantly associated with lower performance in each test, except on MMSE. 
Regarding cognitive change over time, we did not find any longitudinal association between chronic benzodiazepine use and the acceleration of latent cognitive decline (a) in both the crude and adjusted models (respectively benzodiazepines $*$ time $=-0.06 \mathrm{p}$-value $=0.11$ and benzodiazepines $*$ time $=0.010 \mathrm{p}$-value $=0.812$ ). Consistent with this result, we did not find any longitudinal test-specific effect (part (b) of table 2), or overall longitudinal effect (c) for each test (figure 4) regarding the link between chronic use and cognitive decline. Sensitivity analyses adjusted for the anxiety score led to the same conclusions (data not shown).

When investigating the effect of benzodiazepines by separating sedatives from anxiolytics using ATC codes (table 3), after adjustment we found a significant lowering of the latent cognitive level for chronic users of both classes of benzodiazepines, with a greater effect observed in chronic users of anxiolytics. However, we did not find any association with accelerated latent cognitive decline for these two classes. 


\section{DISCUSSION}

The present study, carried out on a large, general elderly population followed-up over 7 years, focused on investigating the impact of chronic benzodiazepine use on cognitive trajectories, by measuring cognition with a wide range of neuropsychological tests, and by modeling a common latent cognitive process. This study emphasized predominant impairment of cognitive level in chronic benzodiazepine users regarding their immediate visual memory and visuomotor processing speed, after adjustment for a wide range of socio-demographic, health behavior and health status factors. However, our results did not highlight an impact of chronic use on accelerated cognitive decline in this elderly population.

Our results differ from those reported in the EVA Study (Paterniti et al, 2002) (n=1,176 subject aged from 60 to 70). In this study, subjects who reported benzodiazepines use in three consecutive examinations over the 4-year follow up presented an accelerated decline of cognitive performance in MMSE, and Digit Symbol Substitution Tests. In the EVA study, subjects were younger than in $\mathrm{E} 3 \mathrm{C}$, fewer (only 80 chronic users), and cognitive decline was analyzed using a dichotomous statistical approach (the outcome considered was the percentage of subjects who declined between the baseline and 4-year follow-up). Our study showed that the sensitivity of some tests is different when detecting cognitive change according to initial cognitive level. Thus, a test like MMSE could have a greater propensity to detect a decline in subjects with a low baseline cognitive level, like chronic users, than in subjects with a higher baseline cognitive level, like non-users. The two other studies carried out on this topic (Dealberto et al, 1997; Lagnaoui et al, 2009) and using the same dichotomous statistical approach, did not find any association with cognitive decline. However, these studies produced little evidence and were subject to methodological limitations (few subjects, only one follow-up point, and no account taken of interference with acute effects).

One major issue when studying drug effects in observational studies is indication bias. Benzodiazepines are frequently prescribed for anxiety and depression, which are both known to 
impair cognitive function. Concerning the cross-sectional association that we found, these and other potential confounders -like age, level of education, etc... - have been accounted for in the multivariate analysis. Moreover, our results were coherent with effects observed in occasional use of benzodiazepine (Buffett-Jerrott et al, 2002; Curran, 1991; Stewart, 2005). However it is always difficult to be sure that a residual confounding effect does not affect our results. Concerning the absence of association with the cognitive decline, the direction of the bias could have led to a false association, but not to the attenuation of a true association with cognitive decline. This bias may be responsible for the statistical trend observed in our crude analysis (beta $=-0.06$ pvalue $=0.10$ ), a trend which is not confirmed in the multivariate analyze, (after adjustment beta $=0.01$ pvalue $=0.81$ ) suggesting its correction by the inclusion in the model of confounders such as anxiety or depression.

Another type of indication bias is the "depletion of susceptible effect" (Lagnaoui et al, 2002; Moride and Abenhaim, 1994). This bias is the consequence of ceasing benzodiazepine prescriptions for subjects with prodromal or incident dementia and may explain the protective effect of benzodiazepines on dementia reported by some studies (Fastbom et al, 1998). It can be illustrated by a recent publication on the discontinuation of psychotropic drugs after initiation of memantine in dementia (Vidal et al, 2008). In the present analysis, we tried to limit this bias by keeping in the analysis subjects who reported a change (cessation or new prescription) in benzodiazepine use with 4 or 7-year follow up, and by considering in the analysis only their cognitive data before changing. We also kept incident cases of dementia with 4 or 7 -year follow up, and in the same way, we censored their cognitive data at the time of diagnosis. This method of selecting subjects also allowed dealing with another type of bias, which was the interference of acute cognitive effect of benzodiazepines in the longitudinal study of cognitive change. Indeed, the real impact on cognitive decline due to chronic benzodiazepine use should be distinguished from the immediate change of cognitive level induced by the initiation or the discontinuation of benzodiazepines and thus by the appearance or disappearance of their acute 
cognitive effects (Dealberto et al, 1997). This interference is also the reason why we had to exclude subjects who reported a change in their benzodiazepine use between baseline and 2 year follow-up (cognitive changes could not be interpreted as a chronic effect of benzodiazepines, or as the initiation or discontinuation of their acute effects).

A potential limitation of our study is the exclusion of some subjects: those who had only one point of follow-up (with no evaluable cognitive decline) and those with missing data. Some cognitive data were also censored to avoid indication bias or interference with acute effect of benzodiazepine (cf. supra). It is always difficult to know the exact influence of these potential selection biases, and such potential diluting factors can complicate the generalization of a lack of difference between groups. However, it seems likely that, if any exists, a major effect of benzodiazepines on cognitive decline would have been detected in spite of these factors.

In our study subjects were categorized on the basis of current use of drugs. Information on the dose and on the treatment history between successive follow-up examinations was not available. A French study carried on elderly (Lechevallier et al, 2003) showed that users who reported taking benzodiazepines at one visit, took it actually every day or almost every day ( $>70 \%$ of users) for at least two years ( $>75 \%$ of users), and in a normal range of recommended doses. Our study defined chronic users from the consumption of benzodiazepines reported at two consecutive visits. Thus it seems reasonable to assume that proportions of daily and/or long-term consumption are higher in our study and that subjects defined as chronic users have a high probability to have been treated between visits. Furthermore, in order to investigate, at least partially, the possibility of a different effect between a daily single low dose to aid sleep and repeated higher doses to manage chronic anxiety, we performed a complementary analysis by distinguishing anxiolytic benzodiazepines, from sedative benzodiazepines. In this analysis, we found a more pronounced cross-sectional lowering of the cognitive performance for subjects taking anxiolytics than for those taking sedatives. However, no such effect was evidenced or suspected on the longitudinal part. 
Our study is the first to propose a quantitative approach for studying the association between benzodiazepine use and cognitive decline. The statistical model handles unbalanced repeated measurements of several bounded quantitative outcomes, and takes into account their metrological properties (Proust et al, 2006). This gave us the opportunity to show that the five tests of the neuropsychological battery had different sensitivities to cognitive changes within the entire range of cognition, and thus that most of these tests (MMSE, TMT, BVRT) should not be interpreted without accounting for their initial value. We were able to take these properties into account to construct a latent cognitive process covering the entire range of cognition to evaluate the effect of benzodiazepines. It is also noteworthy that this latent cognitive process was defined using a set of standardized neuropsychological tests used to assess different domains of cognition (visual memory, verbal fluency, attention, visuomotor processing speed and mental flexibility capacities), and could be interpreted as a global cognitive factor. One of the strengths of this model is that it maximizes the power of the analysis by analyzing simultaneously several neuropsychological tests with different metrological properties. Moreover, it avoids the misspecification of the linear mixed model when evaluating predictors of cognitive decline (Proust-Lima et al, 2011). In consequence, in view of the methodology and of the number of subjects included in our analyses, our study is, to our knowledge, the most powerful analysis conducted on this topic..

The cognitive decline observed in the seven years of our study was highly significant (beta $=-0,125 \mathrm{p}<0.001)$ with a major interaction with age (Interaction $=-0,395 \mathrm{p}=<0.001)$. The rate of decline in our study was of $0.9 \%$ of decline per year at 75 years old and $1.7 \%$ per year at 85 years old. These rates were very similar to those observed in other studies in general population (for review see Park et al, 2003). The improvement of cognitive functioning observed in both groups at the two-year follow up (i.e. variable "first passing effect") was probably due to practice and/or learning effects (Collie et al, 2003). Similarly to some others studies (Collie et al, 2003; Jacqmin-Gadda et al, 1997) the observed improvement was limited to the second testing. 
Although the present paper suggests that long-term benzodiazepine use did not have a significant longitudinal effect on cognitive decline, cross-sectional impairment of cognitive performance is an adverse effect which should not be minimized. Indeed, this impairment could result in the depletion of cognitive reserves and precipitate the onset of dementia, thereby explaining its increased incidence observed in chronic users in some studies (Lagnaoui et al, 2002; Wu et al, 2009). Furthermore, despite the possible recovery of cognitive functions after withdrawal from long-term benzodiazepine use, this recovery after withdrawal could be incomplete with some remaining impairment in some areas of cognition (Barker et al, 2004). Finally other problems associated with prolonged benzodiazepine therapy, such as withdrawal difficulty, dependence or falls and fractures must also be considered in the prescription renewal of benzodiazepines in elderly people (Cumming, 1998; Madhusoodanan and Bogunovic, 2004).

The present study did not provide proof of an absence of effect of benzodiazepines chronic use on cognitive decline. Indeed, in observational studies, the possibility remains that unmeasured confounders or uncontrolled selection biases -due to missing data, death, lost of follow-up- may partly explain the absence of association observed, and this precludes any conclusion of absence of causality. However, the sample size and the duration of the study, the extensive adjustment we made, the robustness of the results after taking into account the potential confounding factors, and the use of an elaborated model constitute strong arguments that increase the likelihood of our observations. These observations suggest that chronic use of benzodiazepines is associated with poorer cognitive performance but not with an accelerated cognitive decline with age. The potential absence of a longitudinal effect of benzodiazepines should not hide the fact that a cross-sectional cognitive effect could exists and persists with chronic use, and that other adverse effects have to be considered in the long-term prescription of benzodiazepines, especially in the elderly.

\section{Bibliography}


Barker MJ, Greenwood KM, Jackson M, Crowe SF (2004). Persistence of cognitive effects after withdrawal from long-term benzodiazepine use: a meta-analysis. Arch Clin Neuropsychol 19(3): 437-454.

Benton A (1965). Manuel pour l'application du test de rétention visuelle. Applications cliniques et expérimentales (in French) Centre de Psychologie appliquée: Paris, France.

Bierman EJ, Comijs HC, Gundy CM, Sonnenberg C, Jonker C, Beekman AT (2007). The effect of chronic benzodiazepine use on cognitive functioning in older persons: good, bad or indifferent? Int J Geriatr Psychiatry 22(12): 1194-1200.

Buffett-Jerrott SE, Stewart SH (2002). Cognitive and sedative effects of benzodiazepine use. Curr Pharm Des 8(1): 45-58.

Cumming RG (1998). Epidemiology of medication-related falls and fractures in the elderly. Drugs \& aging 12(1): 43-53.

Curran HV (1991). Benzodiazepines, memory and mood: a review. Psychopharmacology (Berl) 105(1): $1-8$.

Dealberto MJ, McAvay GJ, Seeman T, Berkman L (1997). Psychotropic drug use and cognitive decline among older men and women. Int J Geriatr Psychiatry 12(5): 567-574.

Fastbom J, Forsell Y, Winblad B (1998). Benzodiazepines may have protective effects against Alzheimer disease. Alzheimer disease and associated disorders 12(1): 14-17.

Folstein MF, Folstein SE, McHugh PR (1975). "Mini-mental state". A practical method for grading the cognitive state of patients for the clinician. J Psychiatr Res 12(3): 189-198.

Food and Drug Administration (1980) Prescribing of minor tranquilizaers. In FDA Drug Bulletin pp 2-3.

führer R, Rouillon F (1989). La version française de l'échelle CES-D (Center for Epidemiologic Studies Depression Scale). Psychiatre et Psychobiologie 4: 163-166.

Galasko DR, Gould RL, Abramson IS, Salmon DP (2000). Measuring cognitive change in a cohort of patients with Alzheimer's disease. Stat Med 19(11-12): 1421-1432.

Hanlon JT, Horner RD, Schmader KE, Fillenbaum GG, Lewis IK, Wall WE, Jr., et al (1998). Benzodiazepine use and cognitive function among community-dwelling elderly. Clinical pharmacology and therapeutics 64(6): 684-692.

Haute Autorité de Santé (2007). Modalités d'arrêt des benzodiazépines et médicaments apparentés chez le patient âgé. recommandation professionnelles 4.

Isaacs B, Kennie AT (1973). The Set test as an aid to the detection of dementia in old people. $\mathrm{Br}$ J Psychiatry 123(575): 467-470.

Jacqmin-Gadda H, Fabrigoule C, Commenges D, Dartigues JF (1997). A 5-year longitudinal study of the Mini-Mental State Examination in normal aging. Am J Epidemiol 145(6): 498-506. 
Lagnaoui R, Begaud B, Moore N, Chaslerie A, Fourrier A, Letenneur L, et al (2002). Benzodiazepine use and risk of dementia: a nested case-control study. Journal of clinical epidemiology 55(3): 314-318.

Lagnaoui R, Tournier M, Moride Y, Wolfson C, Ducruet T, Begaud B, et al (2009). The risk of cognitive impairment in older community-dwelling women after benzodiazepine use. Age and ageing 38(2): 226-228.

Laird NM, Ware JH (1982). Random-effects models for longitudinal data. Biometrics 38(4): 963974.

Lechevallier N, Fourrier A, Berr C (2003). [Benzodiazepine use in the elderly: the EVA Study]. Revue d'epidemiologie et de sante publique 51(3): 317-326.

Madhusoodanan S, Bogunovic OJ (2004). Safety of benzodiazepines in the geriatric population. Expert opinion on drug safety 3(5): 485-493.

Morgan K, Dallosso H, Ebrahim S, Arie T, Fentem PH (1988). Prevalence, frequency, and duration of hypnotic drug use among the elderly living at home. Br Med J (Clin Res Ed) 296(6622): 601-602.

Moride Y, Abenhaim L (1994). Evidence of the depletion of susceptibles effect in nonexperimental pharmacoepidemiologic research. Journal of clinical epidemiology 47(7): 731-737.

National Health Service Grampian (2008) Guidance for prescribing and withrdawal of benzodiazepine and hypnotics in general practice. $\mathrm{p} 1$.

Ohayon MM, Lader MH (2002). Use of psychotropic medication in the general population of France, Germany, Italy, and the United Kingdom. J Clin Psychiatry 63(9): 817-825.

Park HL, O'Connell JE, Thomson RG (2003). A systematic review of cognitive decline in the general elderly population. Int J Geriatr Psychiatry 18(12): 1121-1134.

Paterniti S, Dufouil C, Alperovitch A (2002). Long-term benzodiazepine use and cognitive decline in the elderly: the Epidemiology of Vascular Aging Study. Journal of clinical psychopharmacology 22(3): 285-293.

Proust-Lima C, Amieva H, Dartigues JF, Jacqmin-Gadda H (2007). Sensitivity of four psychometric tests to measure cognitive changes in brain aging-population-based studies. Am $J$ Epidemiol 165(3): 344-350.

Proust-Lima C, Amieva H, Letenneur L, Orgogozo JM, Jacqmin-Gadda H, Dartigues JF (2008). Gender and education impact on brain aging: a general cognitive factor approach. Psychol Aging 23(3): 608-620.

Proust-Lima C, Dartigues JF, Jacqmin-Gadda H (2011). Misuse of the Linear Mixed Model When Evaluating Risk Factors of Cognitive Decline. Am J Epidemiol: [Epub ahead of print].

Proust C, Jacqmin-Gadda H, Taylor JM, Ganiayre J, Commenges D (2006). A nonlinear model with latent process for cognitive evolution using multivariate longitudinal data. Biometrics 62(4): 1014-1024. 
Reitan R (1958). Validity of the Trail Making Test as an indicator of organic brain damage. Perceptual and Motor Skills 8: 271-276.

Ritchie K, Carriere I, de Mendonca A, Portet F, Dartigues JF, Rouaud O, et al (2007). The neuroprotective effects of caffeine: a prospective population study (the Three City Study). Neurology 69(6): 536-545.

Stewart SA (2005). The effects of benzodiazepines on cognition. J Clin Psychiatry 66 Suppl 2: 9-13.

The 3C Study Group (2003). Vascular factors and risk of dementia: design of the Three-City Study and baseline characteristics of the study population. Neuroepidemiology 22(6): 316-325.

Verdoux H, Lagnaoui R, Begaud B (2005). Is benzodiazepine use a risk factor for cognitive decline and dementia? A literature review of epidemiological studies. Psychol Med 35(3): 307315.

Vidal JS, Lacombe JM, Dartigues JF, Pasquier F, Robert P, Tzourio C, et al (2008). Evaluation of the impact of memantine treatment initiation on psychotropics use: a study from the French national health care database. Neuroepidemiology 31(3): 193-200.

WHO Collaborating Centre for Drug Statistics Methodology (2000). Guidelines for ATC classification and DDD assignment: oslo.

Wu CS, Wang SC, Chang IS, Lin KM (2009). The association between dementia and long-term use of benzodiazepine in the elderly: nested case-control study using claims data. Am J Geriatr Psychiatry 17(7): 614-620. 
Table 1: Characteristics of benzodiazepines chronic users and non users.

Non users $\mathrm{N}=4226$ Chronic users $\mathrm{N}=969$-value

Sex, Women, $\%$

Age, Mean(sd)

Education

9 to 12 years, $\%$

12 years or more, $\%$

\section{Center}

Montpellier, \%

Bordeaux, \%

Dijon, $\%$

Occupational status status,

High, \%

\section{Tobacco}

(current or former smoker), \%

\section{Alcohol}

No drinker\%

moderate $\%$

Abuse (>21g H; >14g F)\%

Physical activity

once a week or more\%

\section{Glycaemia}

Impaired Fasting Glucose $\%$

Type 2 Diabetes \%

Hypercholesterolemia, \%

ApoE4, \%

High blood pressure, $\%$

Cardio-vascular history, $\%$

Insomnia, \%

Depressive symptoms, $\%$

High Anxiety*, \%

Baseline neuropsychological scores

MMSE, Mean(sd)

BVRT, Mean(sd)

IST, Mean(sd)

TMT A**, Mean(sd)

TMT B**, Mean(sd)

Anxiety**, \%

56.0

$73.2(5.0)$

29.6

40.9

26.7

18.8

54.4

50.0

40.5

18.7

62.9

18.4

37.2

3.8

8.5

36.1

19.9

57.7

7.7

14.5

13.7

20.7

$27.6(1.8)$

11.8 (1.9)

$31.9(5.2)$

5.1 (1.7)

2.4 (1.2)

\section{9}

$74.6(5.4)$

33.8

32.1

19.5

21.9

58.6

35.0

$<0.001$

29.2

$<0.001$

23.6

$<0.001$

62.1

14.2

24.2

$<0.001$

3.1

0.595

8.5

42.8

$<0.001$

17.3

0.064

62.2

0.010

10.8

0.002

56.9

$<0.001$

40.7

$<0.001$

39.4

$<0.001$

39.4

$27.3(1.9)$

$11.2(2.0)$

$<0.001$

30.5 (5.6)

$<0.001$

$<0.001$

4.5 (1.5)

$<0.001$

$2.0(1.1)$

20.7 $<0.001$

* assessed by the Spielberger inventory trait (we used the $75^{\text {th }}$ percentile as cut off) on 3426 non users and 735 chronics users of Bzd.

**TMTA and TMTB: average number of good displacements in 10 seconds. 
Table 2: Effect of Chronic benzodiazepines use on (a) the latent cognitive process and its change with time in the study and on (b) each of the neuropsychological tests through contrasts

\begin{tabular}{|c|c|c|c|c|c|c|c|}
\hline \multirow{2}{*}{\multicolumn{2}{|c|}{ Variable }} & \multicolumn{3}{|c|}{ Crude model $(\mathrm{N}=5195)$} & \multicolumn{3}{|c|}{ Adjusted model ${ }^{\ddagger}(\mathrm{N}=5195)$} \\
\hline & & Estimate & SE & P-value & Estimate & SE & P-value \\
\hline \multicolumn{8}{|c|}{ Effect of the Covariate on latent cognitive process } \\
\hline \multirow{7}{*}{ (a) } & Intercept & 62,676 & 0,543 & & 57,497 & 0,587 & \\
\hline & Time $^{*}$ & $-0,125$ & 0,029 & $<0,001$ & $-0,290$ & 0,074 & $<0,001$ \\
\hline & First passing effect & $-1,832$ & 0,102 & $<0,001$ & $-1,926$ & 0,102 & $<0,001$ \\
\hline & $\mathrm{Age}^{\dagger}$ at inclusion & $-3,687$ & 0,194 & $<0,001$ & $-3,417$ & 0,182 & $<0,001$ \\
\hline & Age $^{\dagger}$ at inclusion*Time ${ }^{*}$ & $-0,395$ & 0,029 & $<0,001$ & $-0,356$ & 0,029 & $<0,001$ \\
\hline & $\begin{array}{l}\text { Cross-sectional effect } \\
\text { Benzodiazepines }\end{array}$ & $-2,508$ & 0,247 & $<0,001$ & $-1,789$ & 0,248 & $<0,001$ \\
\hline & $\begin{array}{l}\text { Longitudinal effect } \\
\text { Benzodiazepines * Time }^{*}\end{array}$ & $-0,065$ & 0,041 & 0,106 & 0,010 & 0,044 & 0,812 \\
\hline \multicolumn{8}{|c|}{ Cross-sectional test specific effect for benzodiazepines } \\
\hline \multirow{5}{*}{ (b) } & IST & 0,188 & 0,261 & 0,471 & 0,245 & 0,257 & 0,340 \\
\hline & BVRT & $-0,875$ & 0,314 & 0,005 & $-0,838$ & 0,320 & 0,009 \\
\hline & TMTA & $-0,467$ & 0,264 & 0,077 & $-0,618$ & 0,284 & 0,030 \\
\hline & TMTB & 0,079 & 0,227 & 0,727 & 0,061 & 0,306 & 0,843 \\
\hline & MMSE & 1,075 & 0,288 & $<0,001$ & 1,150 & 0,283 & $<0,001$ \\
\hline \multicolumn{8}{|c|}{ Longitudinal test specific effect for benzodiazepines*Time } \\
\hline \multirow{5}{*}{ (b) } & IST & 0,057 & 0,053 & 0,282 & 0,057 & 0,053 & 0,288 \\
\hline & BVRT & 0,119 & 0,084 & 0,158 & 0,120 & 0,093 & 0,197 \\
\hline & TMTA & $-0,108$ & 0,059 & 0,069 & $-0,114$ & 0,066 & 0,082 \\
\hline & TMTB & 0,003 & 0,070 & 0,970 & 0,005 & 0,129 & 0,972 \\
\hline & MMSE & $-0,071$ & 0,069 & 0,305 & $-0,067$ & 0,068 & 0,324 \\
\hline
\end{tabular}

Results from nonlinear mixed models with latent process.

${ }^{*}$ Time variable represents the number of years after inclusion.

†Age at inclusion was included in the model as decades after 65 years old: (age-65)/10;

tEstimates adjusted for center, gender, education, socio-professional status, depression, insomnia, high blood pressure, hypercholesterolemia,

tobacco, alcohol and sports activity. 
Table 3: Impact of chronic benzodiazepines use on the latent cognitive

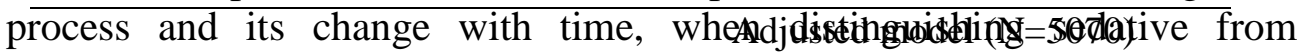
anxiolytic classes of BZD.

Estimate $^{\ddagger} \quad \mathrm{SE}^{\ddagger} \quad \mathrm{P}$-value

Effect of the Covariate on latent cognitive process

\begin{tabular}{llll} 
Intercept & 56,960 & 0,584 & \\
Time $^{*}$ & $-0,331$ & 0,064 & $<0,001$ \\
First passing effect & $-1,978$ & 0,105 & $<0,001$ \\
Age $^{\dagger}$ at inclusion & $-3,514$ & 0,186 & $<0,001$ \\
Age $^{\dagger}$ at inclusion*Time & $-0,360$ & 0,029 & $<0,001$ \\
$\begin{array}{l}\text { Cross-sectional } \\
\begin{array}{l}\text { Sedative } \\
\text { Longitudinal }\end{array}\end{array}$ & $-1,007$ & 0,470 & 0,031 \\
$\begin{array}{l}\text { Sedative *Time } \\
\text { Cross-sectional }\end{array}$ & $-0,028$ & 0,100 & 0,779 \\
$\begin{array}{l}\text { Anxiolytic } \\
\begin{array}{l}\text { Longitudinal } \\
\text { Anxiolytic *Time }\end{array}\end{array}$ & $-1,854$ & 0,298 & $<0,001$ \\
& 0,069 & 0,055 & 0,209 \\
\hline
\end{tabular}

Results from a nonlinear mixed model with a latent process.

${ }^{*}$ Time variable represents the number of years after inclusion.

$\dagger$ Age at inclusion was included in the model as decades after 65 years old: (age-65)/10;

Estimates adjusted for center, gender, education, socio-professional status, depression, insomnia, high blood pressure, hypercholesterolemia, tobacco, alcohol and sports activity. 
Figure 1: Flow chart diagram mapping the selection of the 5195 3C Study participants included in the present analyses

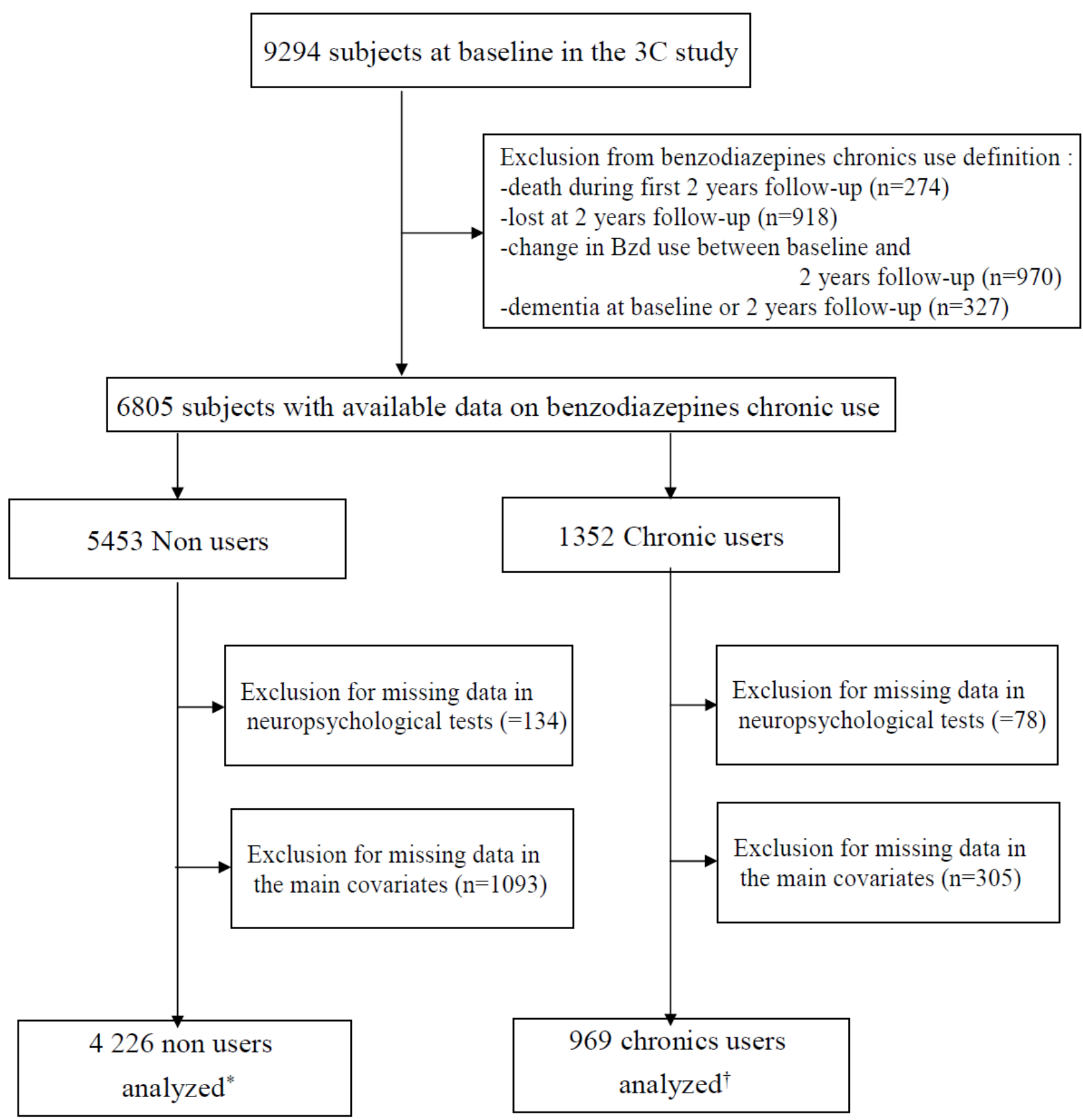

*Among the non-users, cognitive follow-up was censored after the 2-year follow-up for 654 subjects (15.5\%) and after the 4-year follow-up for $970(22.9 \%)$.

$\dagger$ Among the chronic users, cognitive follow-up was censored after the 2-year follow-up for 273 subjects (28.2\%) and after the 4-year follow-up for $312(32.2 \%)$. 
Figure 2: Conceptualisation of the Nonlinear mixed model with a latent process modelling global cognition from 5 neuropsychological tests.

(c)

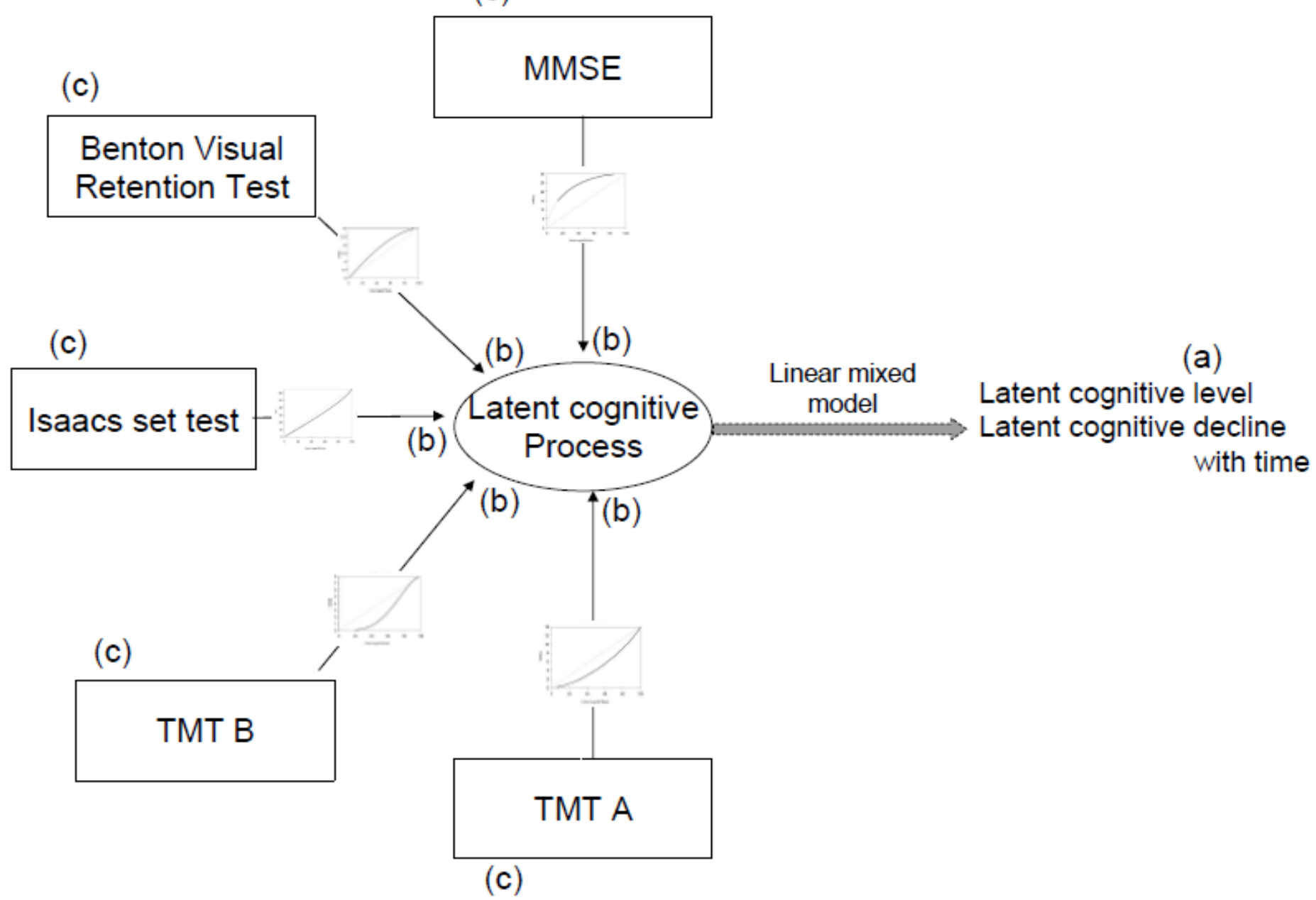

(a) a linear mixed model describes change over time in the latent cognitive process and evaluates the global effect of covariates on this latent cognitive trajectory

(b) test-specific measurement nonlinear models relates each administration of the neuropsychological tests with the latent cognitive process, and evaluates specific effects of the covariates on each test in addition to their global effect on the latent cognitive process.

(c) the overall effect of a covariate on each specific test is calculated by adding together the global (a) and the test-specific effect (b) of the covariate. 
Figure 3: Estimated beta transformations between each neuropsychological test and the latent cognitive process.
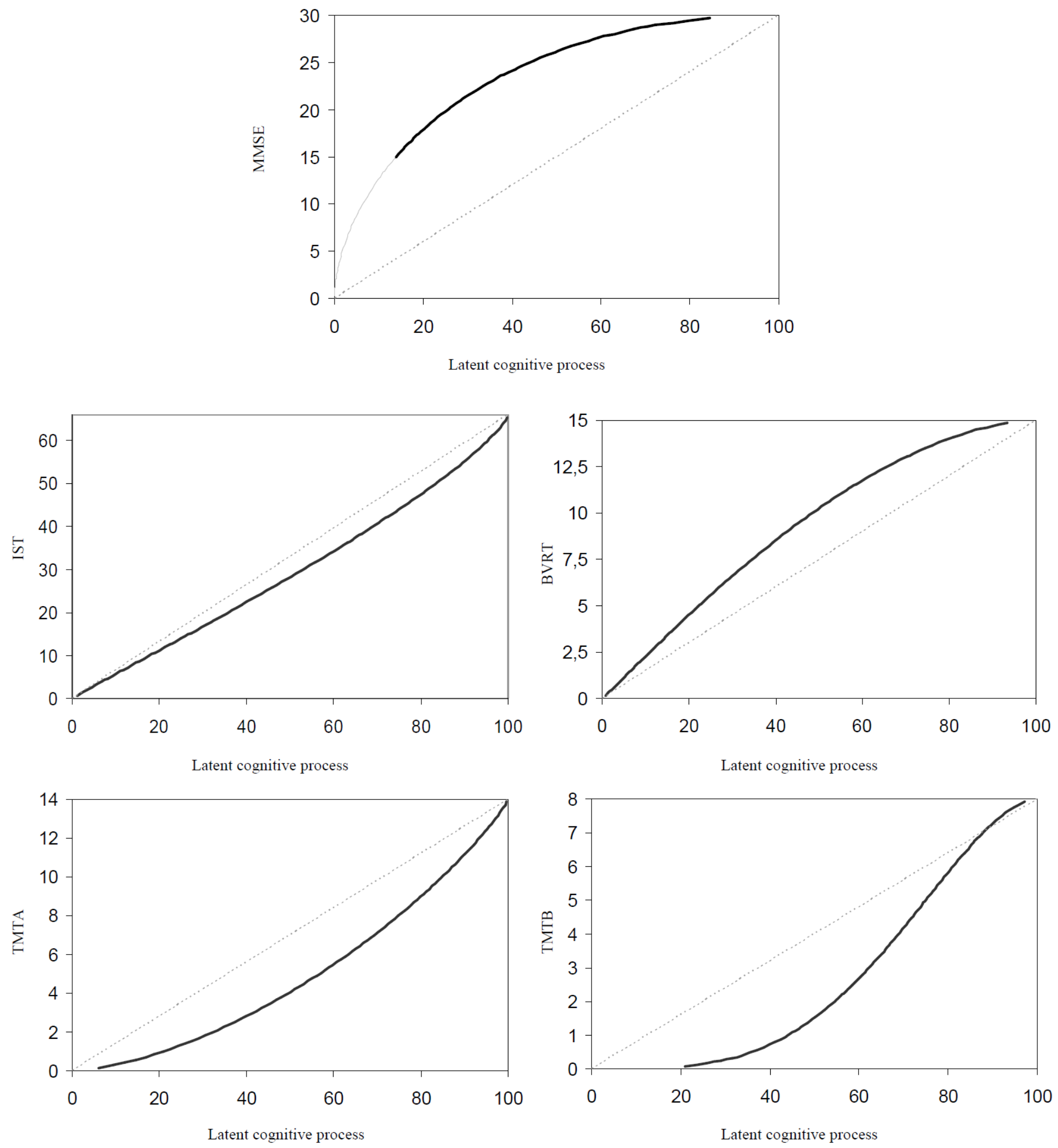
Figure 4: Overall effect (c) of benzodiazepines (Bzd) chronic use on each specific neuropsychological test and predicted mean trajectories after inclusion
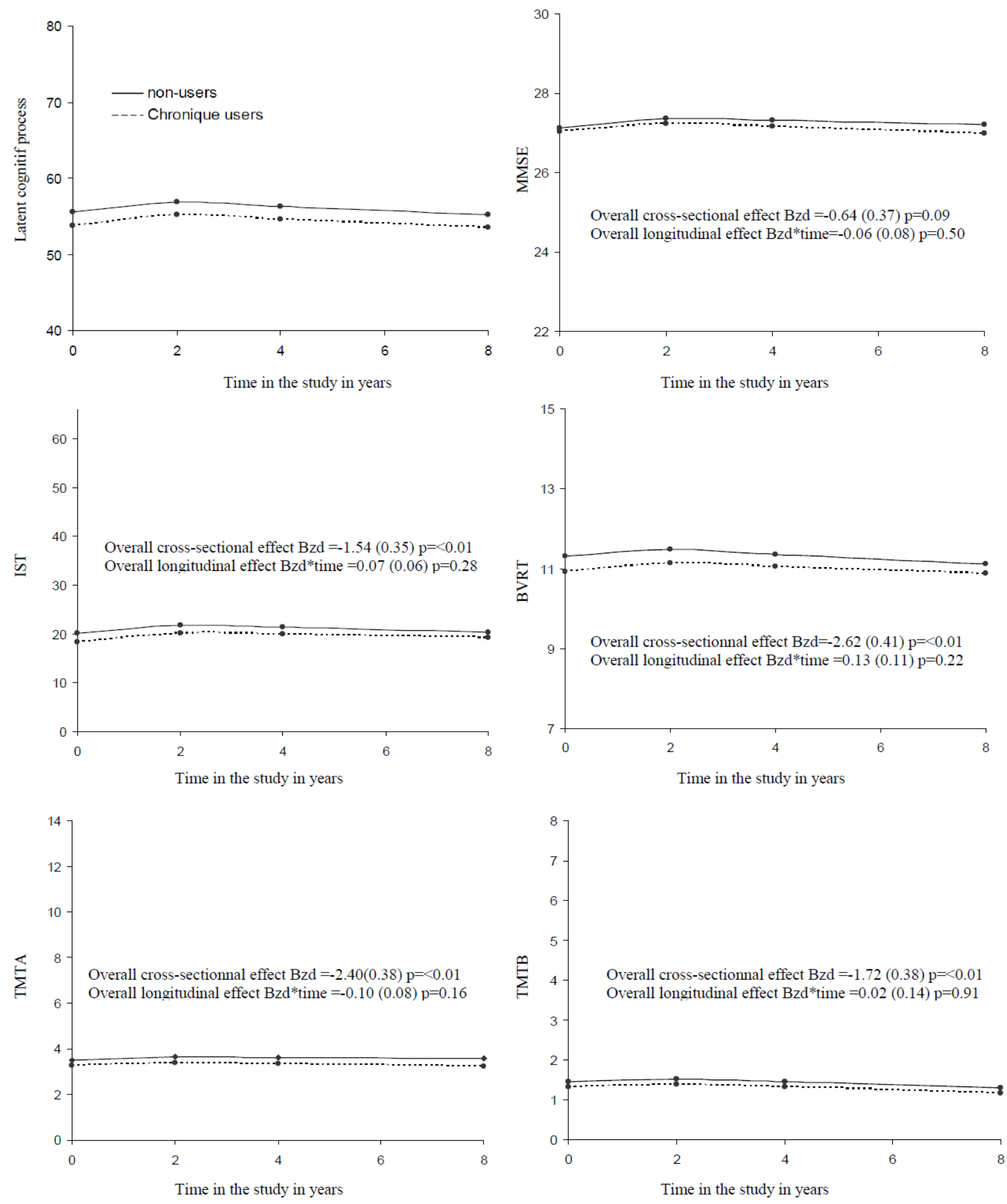

Predicted mean trajectories, including a first passing effect, for a women aged of 65 years old, recruited in Bordeaux, with a level of education below 9 years, a low socio-professional 
status, no hypercholesterolemia, no insomnia, no depression, no alcohol abuse and no physical activity. Time variable represents the number of years after inclusion. 